\title{
Author Correction: Exercise hormone irisin is a critical regulator of cognitive function
}

Mohammad R. Islam, Sophia Valaris, Michael F. Young, Erin B. Haley, Renhao Luo, Sabrina F. Bond, Sofia Mazuera, Robert R. Kitchen (10, Barbara J. Caldarone, Luis E. B. Bettio, Brian R. Christie (D), Angela B. Schmider, Roy J. Soberman, Antoine Besnard (D), Mark P. Jedrychowski, Hyeonwoo Kim, Hua Tu, Eunhee Kim, Se Hoon Choi, Rudolph E. Tanzi (D), Bruce M. Spiegelman (D) and Christiane D. Wrann (D)

Correction to: Nature Metabolism https://doi.org/10.1038/s42255-021-00438-z, published online 20 August 2021

In the version of this Article initially published, while reorganizing data presentation, an oversight led to the panel in Extended Data Fig. $7 \mathrm{j}$ being published as a duplicate of Fig. $6 \mathrm{i}$ in the main text. The correct Extended Data Fig. $7 \mathrm{j}$ has now replaced the original version. This change does not affect the conclusions in the paper.

This change has been made to the online version of this Article.

Published online: 7 October 2021

https://doi.org/10.1038/s42255-021-00476-7

(c) The Author(s), under exclusive licence to Springer Nature Limited 2021 